\title{
Cercarial Dermatitis
}

National Cancer Institute

\section{Source}

National Cancer Institute. Cercarial Dermatitis. NCI Thesaurus. Code C128349.

A pruritic rash that occurs as consequence of cercariae penetration of the skin after aquatic exposure to animal (usually avian) schistosomes, often in countries non-endemic to human schistosomiasis. The condition is non-invasive and responsive to symptomatic treatment. 